\title{
On massive spin 2 interactions
}

\author{
Yu. M. Zinoviev * \\ Institute for High Energy Physics \\ Protvino, Moscow Region, 142280, Russia
}

\begin{abstract}
In this paper we use a constructive approach based on gauge invariant description of massive high spin particles for investigation of possible interactions of massive spin 2 particle. We work with general case of massive spin 2 particle living in constant curvature $(A) d S_{d}$ background, which allows us carefully consider all flat space, massless or partially massless limits. In the linear approximation (cubic terms with no more than two derivatives in the Lagrangians and linear terms with no more than one derivative in gauge transformations) we investigate possible self-interaction, interaction with matter (i.e. spin 0,1 and $1 / 2$ particles) and interaction with gravity.
\end{abstract}

\footnotetext{
*E-mail address: yurii.zinoviev@ihep.ru
} 


\section{Introduction}

In all investigations of massless particles interactions gauge invariance plays a crucial role. Not only it determines a kinematic structure of free theory and guarantees a right number of physical degrees of freedom, but also to a large extent fixes possible interactions of such particles. This leads, in particular, to formulation of so called constructive approach for investigation of massless particles models [1, 2, 3, 4, 5]. In such approach, starting with appropriate collection of free massless particles and requiring conservation of (modified) gauge invariance in the process of switching on an interaction, one can consistently reproduce such physically important theories as Yang-Mills, gravity and supergravity.

Usual description of massive particles does not include gauge invariance and it is hard to formulate one simple principle for constructing consistent theories with such particles. A lot of different requirements, such as conservation of right number of physical degrees of freedom, smooth massless limit, tree level unitarity and causality, was used in the past [6, 17, 8, 9, 10, 11, 12, 13.

There exist two classes of consistent models for massive high spin particles, namely, for massive non-abelian spin 1 particles and for massive spin $3 / 2$ ones. In both cases masses of gauge fields appear as a result of spontaneous gauge symmetry breaking. One of the main ingredients of this mechanism is the appearance of Goldstone particles with non-homogeneous gauge transformations. This, in turn, leads to the gauge invariant description of such massive spin 1 and spin 3/2 particles. But such gauge invariant description of massive particles could be constructed for higher spins as well, e.g. [14, 15, 16, 17, 18, 19, 20, 21, 22, 23. This allows one to extend the above mentioned constructive approach to the case of massive high spin particles.

In the first section of our paper we give two simple examples of such constructive approach. One of them deals with triplet of massive spin 1 particles with gauge group $S U(2) \simeq O(3)$. We show that constructive approach allows one to reproduce two well known possibilities: one based on the non-linear $\sigma$-model [24], and, with the help of introduction of additional scalar field, usual model of spontaneous symmetry breaking with the doublet of Higgs fields. Another example devoted to electromagnetic interaction for massive spin $3 / 2$ particle [9, 10]. In the first linear approximation (i.e. cubic terms in the Lagrangian and linear terms in the gauge transformations) we construct the most general gauge invariant Lagrangian. Our results unambiguously show that any model with minimal gauge interaction of massive spin 3/2 particle in flat space must be a part of some (spontaneously broken) supergravity theory.

Main part of our paper devoted to massive spin 2 particle interactions. It is an old but still unsolved problem and the main difficulty is connected with the so called sixth degree of freedom [25]. Indeed, for the free particle it is easy to choose a structure of mass terms so that there are exactly five degrees of freedom in the model. But in the interacting theory this dangerous (because it is a ghost) sixth degree of freedom reappear. Note that it is, in principle, possible that in the full non-linear theory this DoF become physical [26, 27], but in what follows we will not consider such possibility. One more difficulty that appears in such theories is the absence of smooth massless limit [28, 29]. Moreover, when one considers such theory in a background space with non-zero cosmological term (de Sitter or anti de Sitter spaces) one faces an ambiguity between flat space and massless limits [30, 31, 32]. 
Here we use constructive approach based on the gauge invariant description of massive particles for the investigation of possible massive spin 2 interactions. It turns out that some results depends on the dimensionality of space-time as well as on the presence (or absence) of cosmological term. So we start in section 2 with the gauge invariant formulation of free massive spin 2 particle in the space-time of arbitrary $d>2$ dimension with nonzero cosmological term, that could be positive or negative. This includes, in particular, the so called partially massless spin 2 particle, which could exist in the de Sitter space only [33, 34, 35, 16, 36].

Then, in section 3 we consider possible self-interaction of such particles in linear approximation. We will not make any suggestion about gauge algebra which could stand behind such theory nor we will not insist on any possible geometrical interpretation. Instead, we use a "brute force" method for our construction. Namely, we write the most general Lorentz invariant cubic vertexes in the Lagrangian as well as the most general ansatz for gauge transformations linear in fields and require that the Lagrangian will be gauge invariant. The only restriction (besides Lorentz invariance) we impose is the restriction on the number of derivatives. In this paper we consider only interactions with no more than two derivatives (and gauge transformations with no more than one derivative) leaving investigation of possible higher derivatives interactions for the future.

Clearly, the existence of linear approximation does not guarantee that the construction of full non-linear theory is possible because obstruction can appear in the next approximations. Nevertheless, the investigation of linear approximation is a very important step, because structure of this approximation (and its whole existence) does not depend on the presence of any other field in the theory. Only in the next quadratic approximation one faces the problem that the closure of gauge algebra requires the presence of right (finite or infinite) collection of fields. Thus, the results obtained in our paper are essentially model independent (up to restriction on the number of derivatives).

For the generic values of mass and cosmological constant the linear approximation for self-interaction exists in any space-time dimension $d \geq 3$, but for partially massless spin 2 particle it exists in $d=4$ dimensions only. This clearly related with the fact that only in $d=4$ partially massless theories are conformally invariant [37. One of the non-trivial results is that the structure of gauge transformations for Goldstone field $A_{\mu}$ turns out to be non-canonical, so if one tries to interpret (as usual in gravity) the $\xi_{\mu}$ gauge transformations as general coordinate ones, then this field should give some non-linear realization of this transformations. One more non-trivial result is that two gauge transformations - vector $\xi_{\mu}$ and scalar $\lambda$ ones, do not commute when the mass $m \neq 0$ forming an algebra similar to Weyl one.

In the next section we consider possible cubic couplings of massive spin two particles with matter. Namely, we consider interaction with (massive or massless) spin 0 , spin 1 and spin $1 / 2$ particles. In all cases (except massless spin 1 in $d=4$ ) our results show an ambiguity between flat and massless limits, which shows itself through non-trivial dependence of coupling constant for scalar Goldstone field $\varphi$ and matter fields interactions on mass and cosmological constant. As a result, partially massless spin 2 particle can interact with matter having traceless energy-momentum tensor only, the most important example being the coupling of partially massless spin 2 with massless spin 1 .

Our last section deals with possible interactions for massive spin 2 particles with gravity. 
As is well known, it is impossible to construct non-trivial consistent interacting theory for collection of massless spin 2 particles [38, 39, 40, 41, 42, but it still leaves the possibility to construct non-trivial interaction of gravity with (one or many) massive spin 2 particles. Indeed, such possibility attend much interest recently in very different contexts. First of all, we show that it is impossible to construct cubic vertex with two massless and one massive spin 2 particles while vertex with two massive and one massless ones does exist. Moreover, it is easy to construct general covariant vertex with two massive fields and arbitrary number of massless gravitons. As for the interaction of partially massless spin 2 particles with gravity, no restrictions on the space-time dimension arise in this approximation.

\section{Toy models}

In this section we present two simple examples of constructive approach to massive high spin particles interactions. First one deals with the triplet of massive spin 1 particles, while second one devoted to possible gauge interactions for massive spin $3 / 2$ particle. Results of this section where already known before, but it is instructive to see how they can be reproduced using gauge invariant formulation alone.

\section{$1.1 \quad$ Spin 1}

In this subsection we illustrate our approach by the simplest non-abelian $S U(2) \sim O(3)$ gauge theory with triplet of vector fields $A_{\mu}{ }^{a}, a=1,2,3$. It is well known that with the help of triplet scalar Goldstone fields $\varphi^{a}$ one can easily construct gauge invariant description of free massive particles. In this, the Lagrangian and gauge transformations look like:

$$
\begin{aligned}
\mathcal{L}_{0} & =-\frac{1}{4}\left(A_{\mu \nu}{ }^{a}\right)^{2}+\frac{m^{2}}{2}\left(A_{\mu}{ }^{a}\right)^{2}-m A_{\mu}{ }^{a} \partial_{\mu} \varphi^{a}+\frac{1}{2}\left(\partial_{\mu} \varphi^{a}\right)^{2} \\
\delta_{0} A_{\mu}{ }^{a} & =\partial_{\mu} \xi^{a}, \quad \delta_{0} \varphi^{a}=m \xi^{a}
\end{aligned}
$$

Now, if we switch on usual non-abelian gauge interaction:

$$
\begin{aligned}
A_{\mu \nu}{ }^{a} & =\partial_{\mu} A_{\nu}{ }^{a}-g \varepsilon^{a b c} A_{\mu}{ }^{b} A_{\nu}{ }^{c}-(\mu \leftrightarrow \nu) \\
\delta_{0} A_{\mu}{ }^{a} & =\partial_{\mu} \xi^{a}-g \varepsilon^{a b c} A_{\mu}{ }^{b} \xi^{c}
\end{aligned}
$$

then the Lagrangian will not be gauge invariant any more:

$$
\delta_{0} \mathcal{L}_{0}=-g m \varepsilon^{a b c} A_{\mu}{ }^{a} \partial_{\mu} \varphi^{b} \xi^{c}
$$

but at the linear approximation the gauge invariance could be easily restored with additional vertex in the Lagrangian and appropriate corrections to gauge transformations:

$$
\mathcal{L}_{1}=a_{1} \varepsilon^{a b c} A_{\mu}^{a} \varphi^{b} \partial_{\mu} \varphi^{c}, \quad \delta_{1} \varphi^{a}=g_{1} \varepsilon^{a b c} \varphi^{b} \xi^{c}
$$

provided $a_{1}=-g / 2, g_{1}=-g / 2$ !. Note, that non-canonical value $g / 2$ in the gauge transformations of scalar fields $\varphi^{a}$ shows that these fields do not transform under linear triplet representation of gauge group. As we already mentioned in the introduction, this first step 
does not depend on the presence of any other fields in the model. Now we can proceed in at least two different ways. At first, we can evade introduction of any additional fields and go on by introducing into the Lagrangian terms of the type $A_{\mu} \varphi^{2} \partial_{\mu} \varphi, \varphi^{2}\left(\partial_{\mu} \varphi\right)^{2}$ and so on. It would lead us to the essentially non-linear theory with the Lagrangian [24]:

$$
\mathcal{L}=-\frac{1}{4}\left(A_{\mu \nu}{ }^{a}\right)^{2}+\frac{m^{2}}{2}\left(A_{\mu}{ }^{a}\right)^{2}-m A_{\mu}{ }^{a} E^{a k}(\varphi) \partial_{\mu} \varphi^{k}+\frac{1}{2} g^{k l}(\varphi) \partial_{\mu} \varphi^{k} \partial_{\mu} \varphi^{l}
$$

and gauge transformations for scalar fields:

$$
\delta \varphi^{k}=m\left(E^{-1}\right)^{k a} \xi^{a}
$$

where $g^{k l}=E^{a k} E^{a l}$. This Lagrangian will be gauge invariant provided the "triad" $E^{a k}$ satisfy the equation

$$
\frac{\partial E^{a k}}{\partial \varphi^{l}}-\frac{\partial E^{a l}}{\partial \varphi^{k}}=g \varepsilon^{a b c} E^{b k} E^{c l}
$$

It is easy to check that non-canonical value $g / 2$ in the linear approximation for the $\varphi^{a}$ gauge transformations is a direct consequence of this equation.

But there is another way. We can introduce one more scalar field $\chi$ and try to stop iterations at some order. And indeed, if we add to the Lagrangian of linear approximation all possible cubic and quartic terms with new field:

$$
\mathcal{L}_{2}=\frac{1}{2}\left(\partial_{\mu} \chi\right)^{2}+a_{2} \chi(\partial A)^{a} \varphi^{a}+a_{3} \chi A_{\mu}{ }^{a} \partial_{\mu} \varphi^{a}+a_{4} m \chi\left(A_{\mu}{ }^{a}\right)^{2}+a_{5}\left(A_{\mu}{ }^{a}\right)^{2} \varphi^{2}+a_{6} \chi^{2}\left(A_{\mu}{ }^{a}\right)^{2}
$$

as well as appropriate corrections to the gauge transformations:

$$
\delta \chi=g_{2}(\varphi \xi), \quad \delta_{2} \varphi^{a}=g_{3} \chi \xi^{a}
$$

we can obtain full gauge invariant theory, provided:

$$
g_{2}=a_{2}=\frac{g}{2}, \quad a_{3}=g, \quad g_{3}=a_{4}=-\frac{g}{2}, \quad a_{5}=a_{6}=\frac{g^{2}}{8}
$$

Moreover, if we introduce shifted variable $\tilde{\chi}=\chi-\frac{2 m}{g}$, then total Lagrangian could be rewritten in a familiar form:

$$
\begin{aligned}
\mathcal{L}= & -\frac{1}{4}\left(A_{\mu \nu}{ }^{a}\right)^{2}+\frac{1}{2}\left(\partial_{\mu} \varphi^{a}\right)^{2}+\frac{1}{2}\left(\partial_{\mu} \chi\right)^{2}-\frac{g}{2} \varepsilon^{a b c} A_{\mu}{ }^{a} \varphi^{b} \partial_{\mu} \varphi^{c}+ \\
& +\frac{g}{2} A_{\mu}{ }^{a}\left(\tilde{\chi} \partial_{\mu} \varphi^{a}-\partial_{\mu} \tilde{\chi} \varphi^{a}\right)+\frac{g^{2}}{8}\left(A_{\mu}{ }^{a}\right)^{2}\left(\varphi^{2}+\tilde{\chi}^{2}\right)
\end{aligned}
$$

Up to the arbitrary potential depending on the invariant combination $\varphi^{2}+\tilde{\chi}^{2}$ only this is just the well known model for spontaneous gauge symmetry breaking through the Higgs mechanism. In this, four scalar fields $\varphi^{a}$ and $\chi$ are transformed under the linear doublet representation of gauge group.

Note, that one more possibility arises when one consider systems with infinite number of gauge fields such as so called Kaluza-Klein models e.g. [43, 44, 45, 46, 47, 48]. Indeed, let us consider simplest example - five-dimensional Yang-Mills theory with gauge fields $A_{\mu}{ }^{a}$, 
where $M=0,1,2,3,5$, and some non-abelian gauge algebra $\left[t^{a}, t^{b}\right]=f^{a b c} t^{c}$. Suppose now that one dimension is a compact one, being a circle of radius $R$. From a four-dimensional point of view, such fields represent combinations of vector and scalar ones: $A_{M}\left(x_{\mu}, x_{5}\right) \Rightarrow$ $A_{\mu}\left(x, x_{5}\right), \varphi\left(x, x_{5}\right)$, which equivalent to infinite number of four-dimensional fields:

$$
\begin{aligned}
A_{\mu}{ }^{a}\left(x_{\mu}, x_{5}\right) & =\sum_{n=-\infty}^{n=\infty} A_{\mu(n)}{ }^{a}(x) \exp \left(i M n x_{5}\right) \\
\varphi^{a}\left(x_{\mu}, x_{5}\right) & =\sum_{n=-\infty}^{n=\infty} \varphi_{(n)}{ }^{a}(x) \exp \left(i M n x_{5}\right)
\end{aligned}
$$

where $M \simeq \frac{1}{R}, A_{\mu(n)}^{*}=A_{\mu(-n)}$ and similarly for $\varphi$. Performing integration on $x_{5}$, one obtains four-dimensional gauge theory with infinite dimensional gauge algebra:

$$
\left[t_{n}^{a}, t_{m}^{b}\right]=f^{a b c} t_{n+m}^{c}
$$

In this, gauge transformations of all fields have the form:

$$
\begin{aligned}
\delta A_{\mu(n)}{ }^{a} & =\partial_{\mu} \Lambda_{n}{ }^{a}+f^{a b c} \Lambda_{k}{ }^{b} A_{\mu(n-k)}{ }^{c} \\
\delta \varphi_{n}{ }^{a} & =M n \Lambda_{n}{ }^{a}+f^{a b c} \Lambda_{k}{ }^{b} \varphi_{(n-k)}{ }^{c}
\end{aligned}
$$

From the last line we see, that all the scalar fields, except $\varphi_{0}$, have non-homogeneous transformation laws and play the role of Goldstone fields. As a result, such model describes finite number of massless vector $A_{\mu 0}{ }^{a}$ and scalar $\varphi_{0}{ }^{a}$ fields and infinite tower of massive $n \neq 0$ vector fields. Introducing appropriate covariant derivatives for these fields:

$$
D_{\mu} \varphi_{n}{ }^{a}=\partial_{\mu} \varphi_{n}{ }^{a}-f^{a b c} A_{\mu(k)}^{b} \varphi_{(n-k)}^{c}-M n A_{\mu(n)}^{a}
$$

the total Lagrangian could be written in a compact form:

$$
\mathcal{L}=\sum_{n=-\infty}^{n=\infty}\left[-\frac{1}{4} A_{\mu \nu(n)}{ }^{a} A_{\mu \nu(-n)}{ }^{a}+\frac{1}{2} D_{\mu} \varphi_{n}{ }^{a} D_{\mu} \varphi_{-n}{ }^{a}\right]
$$

More complicated examples, corresponding to partial breaking of initial gauge algebra, could be found in [47].

\subsection{Spin $3 / 2$}

Our second example devoted to the possible electromagnetic interactions for massive spin $3 / 2$ particles [9, 10]. As is well known using two fields - vector-spinor $\Psi_{\mu}$ and spinor $\chi$ one can construct gauge invariant formulation of massive spin $3 / 2$ particle. So we start with the sum of free massive spin $3 / 2$ and massless spin 1 Lagrangians:

$$
\begin{aligned}
\mathcal{L}_{0}= & \frac{i}{2} \varepsilon^{\mu \nu \alpha \beta} \bar{\Psi}_{\mu} \gamma_{5} \gamma_{\nu} \partial_{\alpha} \Psi_{\beta}+\frac{i}{2} \bar{\chi} \gamma^{\mu} \partial_{\mu} \chi-\frac{1}{4} A_{\mu \nu}{ }^{2}- \\
& -\frac{m}{2} \bar{\Psi}_{\mu} \sigma^{\mu \nu} \Psi_{\nu}+i \sqrt{\frac{3}{2}} m(\bar{\Psi} \gamma) \chi-m \bar{\chi} \chi
\end{aligned}
$$


where gauge transformations leaving this Lagrangian invariant look like:

$$
\delta_{0} \Psi_{\mu}=\partial_{\mu} \eta+\frac{i m}{2} \gamma_{\mu} \eta, \quad \delta_{0} \chi=\sqrt{\frac{3}{2}} m \eta, \quad \delta A_{\mu}=\partial_{\mu} \lambda
$$

Here $\eta$ is a spinor parameter, while $\lambda$ is a scalar. We prefer to work with Majorana spinors, so in what follows all spinor objects $\Psi_{\mu}, \chi$ and $\eta$ will be considered as doublets of (anticommuting) Majorana spinors. In this, minimal gauge interactions corresponds to the replacement of ordinary partial derivatives by covariant ones:

$$
\partial_{\mu} \Rightarrow D_{\mu}=\partial_{\mu}+q A_{\mu}, \quad q=\left(\begin{array}{cc}
0 & e \\
-e & 0
\end{array}\right), \quad q^{2}=-e^{2} I
$$

As a result of such replacement, the Lagrangian lost its gauge invariance (just because covariant derivatives do not commute):

$$
\delta_{0} \mathcal{L}_{0}=i \bar{\Psi}_{\mu} q \tilde{A}^{\mu \nu} \gamma_{5} \gamma_{\nu} \eta, \quad \tilde{A}^{\mu \nu}=\frac{1}{2} \varepsilon^{\mu \nu \alpha \beta} A_{\alpha \beta}
$$

So we try to restore gauge invariance by adding to the Lagrangian non-minimal terms linear in gauge field-strength $A_{\mu \nu}$ :

$$
\begin{aligned}
m \mathcal{L}_{1}= & \frac{1}{2} \bar{\psi}_{\mu}\left[a_{1} A^{\mu \nu}+a_{2} \gamma_{5} \tilde{A}^{\mu \nu}+a_{3} g^{\mu \nu}(\sigma A)+a_{4}\left(A^{\mu \alpha} \sigma_{\alpha}{ }^{\nu}+\sigma^{\mu \alpha} A_{\alpha}{ }^{\nu}\right)\right] q \Psi_{\mu}+ \\
& +i \bar{\Psi}_{\mu}\left(a_{5} A^{\mu \nu}+a_{6} \gamma_{5} \tilde{A}^{\mu \nu}\right) \gamma_{\nu} q \chi+\frac{a_{7}}{2} \bar{\chi} q(\sigma A) \chi
\end{aligned}
$$

as well as all possible linear terms for $\eta$-transformations for all three fields:

$$
\begin{aligned}
& m \delta_{1} \Psi_{\mu}=i q\left(\alpha_{1} A_{\mu \nu}+\alpha_{2} \gamma_{5} \tilde{A}_{\mu \nu}\right) \gamma^{\nu} \eta \quad m \delta_{1} \chi=q \alpha_{3}(\sigma A) \eta \\
& m \delta_{1} A_{\mu}=\alpha_{4}\left(\bar{\Psi}_{\mu} q \eta\right)+i \alpha_{5}\left(\bar{\chi} \gamma_{\mu} q \eta\right)
\end{aligned}
$$

Straightforward calculations show that the gauge invariance could be restored (up to terms quadratic in $A_{\mu \nu}$ ) provided all the unknown coefficients are expressed in terms of two parameters, say $\alpha_{1}$ and $\alpha_{3}$,

$$
\begin{aligned}
& \alpha_{2}=\alpha_{1}, \quad \alpha_{4}=2 \alpha_{1}, \quad \alpha_{5}=-2 \alpha_{3}, \quad a_{1}=-2 \alpha_{1}, \\
& a_{2}=2 \alpha_{1} . \quad a_{3}=a_{4}=0, \quad a_{5}=a_{6}=-2 \alpha_{3}, \quad a_{7}=2 \sqrt{\frac{2}{3}} \alpha_{3}
\end{aligned}
$$

while this two parameters satisfy the relation:

$$
2 \alpha_{1}-4 \sqrt{\frac{3}{2}} \alpha_{3}+1=0
$$

So we have one paramater family of Lagrangians. Now, if we calculate the commutator of two $\eta$ transformations on the vector field $A_{\mu}$, we obtain:

$$
\left[\delta_{1}, \delta_{2}\right] A_{\mu}=-4 i e^{2}\left(\alpha_{1}^{2}+2 \alpha_{3}^{2}\right)\left(\overline{\eta_{2}} \gamma^{\nu} \eta_{1}\right) A_{\nu \mu}
$$


Due to relation on the parameters $\alpha_{1}$ and $\alpha_{3}$ it is impossible to set both parameters simultaneously equal to zero. This means that commutator of two $\eta$ transformations always give a translation and to proceed further on we must introduce graviton as well. So any model with minimal gauge interaction for massive spin 3/2 particle in flat space must be a part of some (spontaneously broken) supergravity theory. In this case all coupling constants are related with gravitational one $k \sim \frac{1}{m_{p l}}$ and we obtain usual for supergravity models relation betwen spin 3/2 mass, Plank mass and gauge coupling constant $m \sim e m_{p l}$ ! Note in pass that one more example of constructive approach, namely electromagnetic interactions for massive spin 2 particles, could be found in [15].

\section{Free massive spin 2}

Our main starting point is the gauge invariant description of free massive spin 2 particle. As we will see part of the results obtained crucially depend on the space-time dimension $d$ and/or the presence or absence of cosmological term. So in what follows we will work in space-time with arbitrary $d \geq 3$ dimension and with non-zero (positive or negative) cosmological term. We denote metric of such space as $g_{\mu \nu}$ and appropriate covariant derivatives as $D_{\mu}$. Till the last section, where $g_{\mu \nu}$ will become dynamical, it is just fixed background metric. Our convention for the commutator of two covariant derivatives is:

$$
\left[D_{\mu}, D_{\nu}\right] v_{\alpha}=\kappa\left(g_{\mu \alpha} \delta_{\nu}^{\beta}-\delta_{\mu}{ }^{\beta} g_{\nu \alpha}\right) v_{\beta}
$$

where $\kappa=-\frac{2 \Lambda}{(d-1)(d-2)}, \Lambda$ - cosmological term.

One of the nice features of gauge invariant description is that it allows one to consider general case of non-zero mass and cosmological term including all possible massless and partially massless limits [16]. To describe the massive spin 2 particle in $d$-dimensional constant curvature space we will use the following Lagrangian:

$$
\begin{aligned}
\mathcal{L}_{0}= & \frac{1}{2} D^{\alpha} h^{\mu \nu} D_{\alpha} h_{\mu \nu}-\frac{1}{2} D^{\alpha} h^{\mu \nu} D_{\mu} h_{\nu \alpha}-\frac{1}{2}(D h)^{\mu}(D h)_{\mu}+(D h)^{\mu} D_{\mu} h-\frac{1}{2} D^{\mu} h D_{\mu} h- \\
& -\frac{1}{2}\left(D_{\mu} A_{\nu}-D_{\nu} A_{\mu}\right)^{2}+\frac{2(d-1)}{d-2}\left(D_{\mu} \varphi\right)^{2}+2 m\left(h^{\mu \nu} D_{\mu} A_{\nu}-h(D A)\right)- \\
& -\frac{4(d-1) c_{0}}{d-2} A^{\mu} D_{\mu} \varphi-\frac{m^{2}+\kappa(d-2)}{2}\left(h^{\mu \nu} h_{\mu \nu}-h^{2}\right)- \\
& -\frac{2(d-1) m c_{0}}{d-2} h \varphi+\frac{2 d(d-1) m^{2}}{(d-2)^{2}} \varphi^{2}+2 \kappa(d-1) A_{\mu}{ }^{2}
\end{aligned}
$$

where $c_{0}=\sqrt{m^{2}+\kappa(d-2)}$. Here symmetric tensor $h_{\mu \nu}$ is the main gauge field, while vector $A_{\mu}$ and scalar $\varphi$ are Goldstone fields necessary for gauge invariance. To simplify the calculations we have chosen non-canonical normalization for kinetic terms of these fields. Also note that there is an ambiguity in the structure of kinetic terms for the $h_{\mu \nu}$ field. Indeed, in flat space (where derivatives commute) the second and third terms in the first line differ by total divergence only. But in spaces with non-zero cosmological term they lead to slightly different structure of mass-like terms and the choice we made gives the most simple 
and convenient structure of these terms. It is not hard to check that this Lagrangian is invariant under the following local gauge transformations:

$$
\begin{aligned}
\delta_{0} h_{\mu \nu} & =D_{\mu} \xi_{\nu}+D_{\nu} \xi_{\mu}+\frac{2 m}{d-2} g_{\mu \nu} \lambda \\
\delta_{0} A_{\mu} & =D_{\mu} \lambda+m \xi_{\mu} \quad \delta_{0} \varphi=c_{0} \lambda
\end{aligned}
$$

Let us note, that these and all subsequent results depend crucially on use of canonical description of massless spin 2 particles. As is known [49], there exists alternative possibility based on traceless symmetric tensor (or on additional Weyl symmetry). Canonical analysis shows that right number of physical degrees of freedom in this case is achieved due to combination of first and second class constraints. So the relation between alternative and canonical formulations is similar to that between, say, non gauge invariant and gauge invariant formulations of massive spin 1 particle. Indeed, additional scalar degree of freedom (trace $h$ in this case) promotes second class constraints to the first class ones. As we have already note in the Introduction, it is hard to formulate constructive approach for theories with second class constraints. Moreover, as it was shown in [49], any attempt to give mass to graviton in such alternative formulation unavoidably leads to the appearance of ghosts.

Note also, that there exists a disagreement on the definition of mass in (A)dS space. Indeed, even for the massless particles gauge invariance requires some "mass-like" terms in the Lagrangians to be present and one often tries to interpret these terms as real mass. Gauge invariant description of massive particles gives, above all, simple and unambiguous definition of massless limit, namely it is a limit where Goldstone fields decouple from the main gauge field.

Let us stress the important difference between massless and massive cases for spin 2 particle. In the massless case we have one gauge symmetry with vector parameter $\xi_{\mu}$ only, while for non-zero mass we have two gauge symmetries with vector $\xi_{\mu}$ and scalar $\lambda$ parameters and only these two symmetries together could guarantee the right number of physical degrees of freedom even after switching on an interaction. Thus, models based on the $\xi_{\mu}$ invariance only like those based on $O S P(4)$ symmetry [50] or models exploring $\lambda$ transformations only [51] always require additional checks for consistency. One more example is a BRST construction of [52], where for massive spin 2 particles authors promote gauge transformations with vector parameter only (and introduce vector Goldstone field $u_{\mu}$ only). As a result, such theory describes additional scalar degrees of freedom and all previous results point that it must be ghost. At the same time, BRST construction for pure massive spin 2 particle does exist [18], but requires additional scalar gauge transformations and scalar Goldstone field.

Note also that if one introduces gauge invariant under the $\lambda$ transformations derivatives:

$$
\nabla_{\mu} h_{\alpha \beta}=D_{\mu} h_{\alpha \beta}-\frac{2 m}{d-2} A_{\mu} \eta_{\alpha \beta}, \quad \nabla_{\mu} \varphi=D_{\mu} \varphi-c_{0} A_{\mu}
$$

then the Lagrangian could be rewritten in more simple form:

$$
\begin{aligned}
\mathcal{L}_{0}= & \frac{1}{2} \nabla^{\alpha} h^{\mu \nu} \nabla_{\alpha} h_{\mu \nu}-\frac{1}{2} \nabla^{\alpha} h^{\mu \nu} \nabla_{\mu} h_{\nu \alpha}-\frac{1}{2}(\nabla h)^{\mu}(\nabla h)_{\mu}+(\nabla h)^{\mu} \nabla_{\mu} h- \\
& -\frac{1}{2} \nabla^{\mu} h \nabla_{\mu} h-\frac{1}{2}\left(D_{\mu} A_{\nu}-D_{\nu} A_{\mu}\right)^{2}+\frac{2(d-1)}{d-2}\left(\nabla_{\mu} \varphi\right)^{2}- \\
& -\frac{m^{2}+\kappa(d-2)}{2}\left(h^{\mu \nu} h_{\mu \nu}-h^{2}\right)-\frac{2(d-1) m c_{0}}{d-2} h \varphi+\frac{2 d(d-1) m^{2}}{(d-2)^{2}} \varphi^{2}
\end{aligned}
$$


As could be easily seen from the formulas given above in the massless limit $m \rightarrow 0$ the Lagrangian breaks into two independent parts. One of them (for symmetric tensor $h_{\mu \nu}$ ) gives usual description of massless spin 2 particle in $(A) d S_{d}$ background, while the other one (with vector $A_{\mu}$ and scalar $\varphi$ fields) gives gauge invariant description of massive spin 1 particle (or sum of massless spin 1 and spin 0 particles in a flat case). But in de Sitter space $\kappa<0$ there exist one more special limit $c_{0} \rightarrow 0$. In this, the scalar field $\varphi$ completely decouples, while pair $h_{\mu \nu}, A_{\mu}$ corresponds to the so called partially massless spin 2 particle. Note that one can use $\xi_{\mu}$ gauge transformation in order to set vector $A_{\mu}=0$. In this, the resulting simple Lagrangian for $h_{\mu \nu}$ will still be invariant under the $\lambda$ transformations, provided we supplement it with restoring gauge $A_{\mu}=0$ transformation with $\xi_{\mu}=-\frac{1}{m} D_{\mu} \lambda$ :

$$
\delta h_{\mu \nu}=-\frac{1}{m}\left(D_{\mu} D_{\nu}+D_{\nu} D_{\mu}\right) \lambda+\frac{2 m}{d-2} g_{\mu \nu} \lambda
$$

We have explicitly checked that such gauge fixed Lagrangian is indeed invariant under this transformation.

\section{Self-interaction}

In this section we consider self-interaction of such massive spin 2 particles in linear approximation. As we have already mentioned in the introduction, we will not make any suggestion on the structure of gauge algebra which could stand behind this theory nor we will not insist on any geometrical interpretation. Instead, we will construct the most general Lorentz invariant cubic terms for the Lagrangian as well as the most general ansatz for gauge transformations and require that the Lagrangian will be gauge invariant. As a result this section will be the most technical part of the paper, but it is important to make sure that our results are completely general.

Even for the massless particles in $(A) d S_{d}$ gauge invariance require introduction of masslike terms into the Lagrangian and appropriate corrections to gauge transformation laws so that the structure of the theory resembles that of massive theory in flat space. Thus, working

with general case of massive particles in $(A) d S_{d}$, it is convenient to organize the calculations just by the number of derivatives. So we start here with the Lagrangian terms with two derivatives and gauge transformations with one derivative and analyze all possible vertexes compatible with gauge invariance up to the lower derivatives terms.

$h h h$-vertex. In this case the most general ansatz for gauge transformations linear in $h$-field and containing one derivative looks as follows:

$$
\begin{aligned}
\delta h_{\mu \nu} & =c_{1} \xi^{\alpha} D_{\alpha} h_{\mu \nu}+c_{2}\left((D h)_{\mu} \xi_{\nu}+(D h)_{\nu} \xi_{\mu}\right)+c_{3} g_{\mu \nu}(D h)_{\alpha} \xi^{\alpha}+ \\
& +c_{4} g_{\mu \nu} D_{\alpha} h \xi^{\alpha}+c_{5}\left(D_{\mu} h_{\nu \alpha}+D_{\nu} h_{\mu \alpha}\right) \xi^{\alpha}+c_{6}\left(D_{\mu} h \xi_{\nu}+D_{\nu} h \xi_{\mu}\right)+ \\
& +c_{7}\left(h_{\alpha \mu} D_{\nu} \xi^{\alpha}+h_{\nu \alpha} D_{\mu} \xi^{\alpha}\right)+c_{8} h\left(D_{\mu} \xi_{\nu}+D_{\nu} \xi_{\mu}\right)+c_{9} h_{\mu \nu}(D \xi)+ \\
& +c_{10}\left(h_{\mu}{ }^{\alpha} D_{\alpha} \xi_{\nu}+h_{\nu}{ }^{\alpha} D_{\alpha} \xi_{\mu}\right)+c_{11} g_{\mu \nu} h^{\alpha \beta} D_{\alpha} \xi_{\beta}+c_{12} g_{\mu \nu} h(D \xi)
\end{aligned}
$$

But due to gauge invariance of free Lagrangian the structure of this transformations could be defined only up to arbitrary field dependent gauge transformations (or in other words up to possible redefinitions of $\xi_{\mu}$ parameter) which in this case have the form:

$$
\delta h_{\mu \nu}=d_{1}\left[D_{\mu}\left(h_{\nu \alpha} \xi^{\alpha}\right)+D_{\nu}\left(h_{\mu \alpha} \xi^{\alpha}\right)\right]+d_{2}\left[D_{\mu}\left(h \xi_{\nu}\right)+D_{\nu}\left(h \xi_{\mu}\right)\right]
$$


Moreover, as in all theories where interacting Lagrangian contains the same number of derivatives as the free one, there always exists a family of physically equivalent Lagrangians related by trivial field redefinitions. In the case at hands, we have a possibility to make such redefinition with four arbitrary parameters:

$$
h_{\mu \nu} \Rightarrow h_{\mu \nu}+s_{1} h_{\mu}{ }^{\alpha} h_{\alpha \nu}+s_{2} h h_{\mu \nu}+s_{3} g_{\mu \nu} h_{\alpha \beta}{ }^{2}+s_{4} g_{\mu \nu} h^{2}
$$

We use all these freedom to bring the gauge transformations to the following more simple form:

$$
\begin{aligned}
\delta h_{\mu \nu} & =c_{1} \xi^{\alpha} D_{\alpha} h_{\mu \nu}+c_{2}\left((D h)_{\mu} \xi_{\nu}+(D h)_{\nu} \xi_{\mu}\right)+c_{3} g_{\mu \nu}(D h)_{\alpha} \xi^{\alpha}+ \\
& +c_{4} g_{\mu \nu} D_{\alpha} h \xi^{\alpha}+c_{7}\left(h_{\alpha \mu} D_{\nu} \xi^{\alpha}+h_{\nu \alpha} D_{\mu} \xi^{\alpha}\right)+c_{9} h_{\mu \nu}(D \xi)
\end{aligned}
$$

At last, we require that the algebra of these transformations be closed, i.e.

$$
\left[\delta\left(\xi_{1}\right), \delta\left(\xi_{2}\right)\right]=\delta\left(\xi_{3}\right)
$$

Simple calculations immediately give $c_{2}=c_{3}=c_{4}=c_{9}=0$ and $c_{7}=c_{1}$. In the massless case the only remaining parameter $c_{1}$ corresponds to gravitational coupling constant $k \sim 1 / m_{p l}$. In what follows, we set $c_{1}=2$. Thus, we obtain a very simple final form for these gauge transformations:

$$
\delta h_{\mu \nu}=2\left[\xi^{\alpha} D_{\alpha} h_{\mu \nu}+D_{\mu} \xi^{\alpha} h_{\alpha \nu}+D_{\nu} \xi^{\alpha} h_{\alpha \mu}\right]
$$

In this,

$$
\xi_{3 \mu}=\xi_{2}{ }^{\alpha} D_{\alpha} \xi_{1 \mu}-\xi_{1}{ }^{\alpha} D_{\alpha} \xi_{2 \mu}
$$

Certainly, these transformations look exactly like general coordinate transformations for covariant second rank symmetric tensor, but let us stress that in our case these are just gauge transformations for spin 2 field living in fixed $(A) d S_{d}$ background. Recall also, that concrete form of these transformations (i.e. that of covariant tensor and not that of contravariant one or tensor density) is just a matter of our choice. This and all our results that follows are always defined up to possible field redefinitions of the type shown above.

Now we construct the most general Lorentz invariant cubic terms with two derivatives:

$$
\begin{aligned}
\mathcal{L}_{h h h} & =h^{\mu \nu}\left[a_{1} D_{\mu} h_{\nu \alpha}(D h)^{\alpha}+a_{2} D_{\mu} h_{\nu \alpha} D^{\alpha} h+a_{3} D_{\mu} h_{\alpha \beta} D_{\nu} h^{\alpha \beta}+a_{4} D_{\mu} h D_{\nu} h+\right. \\
& +a_{5} D_{\mu} h^{\alpha \beta} D_{\nu} h_{\alpha \beta}+a_{6} D_{\mu} h(D h)_{\nu}+a_{7} D_{\alpha} h_{\mu \nu}(D h)^{\alpha}+a_{8} D_{\alpha} h_{\mu \nu} D_{\alpha} h+ \\
& \left.+a_{9} D_{\alpha} h_{\beta \mu} D^{\beta} h^{\alpha \nu}+a_{10} D_{\alpha} h_{\beta \mu} D^{\alpha} h^{\beta \nu}+a_{11}(D h)_{\mu}(D h)_{\nu}\right]+h\left[a_{12} D_{\mu} h_{\alpha \beta} D^{\mu} h^{\alpha \beta}+\right. \\
& \left.+a_{13} D_{\mu} h D^{\mu} h+a_{14}(D h)_{\mu}(D h)^{\mu}+a_{15}(D h)_{\mu} D^{\mu} h+a_{16} D_{\mu} h_{\alpha \beta} D^{\alpha} h^{\beta \mu}\right]
\end{aligned}
$$

As in the free case, we face an ambiguity because in a flat space not all these terms are independent, namely up to total divergence we have (schematically):

$$
\begin{aligned}
& \left(a_{1}\right)=\left(a_{5}\right)+\left(a_{9}\right)-\left(a_{11}\right) \\
& \left(a_{2}\right)=\left(a_{6}\right)+\left(a_{14}\right)-\left(a_{16}\right)
\end{aligned}
$$

so there exists a family of equivalent Lagrangians with two arbitrary parameters. In a constant curvature space different choices lead to slightly different structure of mass-like $h^{3}$ 
terms and again we use this freedom to bring these terms to the most simple form. Then the requirement that the Lagrangian will be gauge invariant (up to lower derivative terms given below) gives:

$$
\begin{gathered}
a_{1}=a_{11}=3 / 2, \quad a_{2}=-3 / 2, \quad a_{3}=-1, \quad a_{4}=a_{15}=1, \quad a_{5}=5 / 2, \quad a_{6}=-5 / 2, \\
a_{7}=a_{10}=-2, \quad a_{8}=2, \quad a_{9}=a_{12}=1 / 2, \quad a_{13}=a_{14}=a_{16}=-1 / 2
\end{gathered}
$$

In this, in the non-flat space background we still have non-compensated variations to be taken into account in the lower derivatives orders:

$$
\begin{aligned}
\delta_{0} \mathcal{L}_{h h h}+\delta_{1} \mathcal{L}_{0} & =\kappa\left[(3 d-8) h^{\alpha \beta} D_{\alpha} h_{\beta \mu}-2(2 d-5) h^{\alpha \beta} D_{\mu} h_{\alpha \beta}+2(d-3) h D_{\mu} h-\right. \\
& \left.-2(d-3) h_{\mu}{ }^{\alpha} D_{\alpha} h+(3 d-8) h_{\mu}{ }^{\alpha}(D h)_{\alpha}-2(d-3) h(D h)_{\mu}\right] \xi^{\mu}
\end{aligned}
$$

Till now all calculations are the same for massive as well as massless spin 2 particles. Let us make here a comment on the so called gravity reconstruction, i.e. on possibility to reconstruct full gravity theory starting from free massless spin 2 particle in flat or constant curvature space background [53]. As usual, in all calculations of the type given above, all the variations which are total divergence are dropped out and of course none of such terms can be "reconstructed" in such iterations. But nothing prevent of to add to the Lagrangian any such terms to bring the result in a convenient form. As for the ambiguity which arise in this iterative process and which is often related with ambiguity in the definition of energymomentum tensor for gravity or matter fields, we have seen that this ambiguity clearly related with trivial field redefinitions and up to this freedom the results are essentially unique.

$h A A$-vertex. Here we start with the most general ansatz for $\xi_{\mu}$ transformations:

$$
\begin{aligned}
\delta A_{\mu}= & c_{1} D_{\mu} A_{\nu} \xi^{\nu}+c_{2} D_{\nu} A_{\mu} \xi^{\nu}+c_{3}(D A) \xi_{\mu}+ \\
& +c_{4} A_{\nu} D_{\mu} \xi^{\nu}+c_{5} A^{\nu} D_{\nu} \xi_{\mu}+c_{6} A_{\mu}(D \xi)
\end{aligned}
$$

As in the previous case there exists a freedom connected with the possible redefinitions of field $A_{\mu}$ and parameter $\xi_{\mu}$ :

$$
\delta A_{\mu}=d_{1} D_{\mu}(A \xi) \quad A_{\mu} \rightarrow A_{\mu}+s_{1} h_{\mu \nu} A^{\nu}+s_{2} h A_{\mu}
$$

so without lost of generality we can restrict ourselves to

$$
\delta A_{\mu}=c_{1} D_{\mu} A_{\nu} \xi^{\nu}+c_{2} D_{\nu} A_{\mu} \xi^{\nu}+c_{3}(D A) \xi_{\mu}
$$

Usually, then one considers an interaction of gravity with vector fields (abelian or nonabelian) one assumes that gravitational field $h_{\mu \nu}$ is inert under the gauge transformations of these fields. But now vector field $A_{\mu}$ is just a component of massive spin 2 field, so we have to consider all the possible gauge transformations with $\lambda$ parameter also. Most general form looks like:

$$
\begin{aligned}
\delta h_{\mu \nu} & =c_{4} D_{(\mu} A_{\nu)} \lambda+c_{5} g_{\mu \nu}(D A) \lambda+c_{6} A_{(\mu} D_{\nu)} \lambda+c_{7} g_{\mu \nu} A^{\alpha} D_{\alpha} \lambda \\
\delta A_{\mu} & =c_{8} D_{\mu} h \lambda+c_{9}(D h)_{\mu} \lambda+c_{10} h D_{\mu} \lambda+c_{11} h_{\mu \nu} D^{\nu} \lambda
\end{aligned}
$$


Once again using the freedom that exists here:

$$
\delta h_{\mu \nu}=d_{2} D_{(\mu}\left(A_{\nu)} \lambda\right) \quad \delta A_{\mu}=d_{3} D_{\mu}(h \lambda) \quad h_{\mu \nu} \rightarrow h_{\mu \nu}+s_{3} A_{\mu} A_{\nu}+s_{4} g_{\mu \nu} A_{\alpha}{ }^{2}
$$

we can reduce these transformations to the form:

$$
\begin{aligned}
\delta h_{\mu \nu} & =c_{5} g_{\mu \nu}(D A) \lambda \\
\delta A_{\mu} & =c_{9}(D h)_{\mu} \lambda+c_{10} h D_{\mu} \lambda+c_{11} h_{\mu \nu} D^{\nu} \lambda
\end{aligned}
$$

Now we proceed by constructing the most general Lorentz invariant cubic terms with two derivatives:

$$
\begin{aligned}
\mathcal{L}_{h A A}= & h^{\mu \nu}\left[a_{1} D_{\mu} A_{\nu}(D A)+a_{2} D_{\mu} A^{\alpha} D_{\nu} A_{\alpha}+a_{3} D_{\mu} A^{\alpha} D_{\alpha} A_{\nu}+a_{4} D^{\alpha} A_{\mu} D_{\alpha} A_{\nu}\right]+ \\
& +h\left[a_{5} D^{\alpha} A^{\beta} D_{\alpha} A_{\beta}+a_{6} D^{\alpha} A_{\beta} D_{\beta} A_{\alpha}+a_{7}(D A)(D A)\right]+ \\
& +a_{8} D^{\alpha} h^{\mu \nu} D_{\alpha} A_{\mu} A_{\nu}+a_{9}(D h)^{\mu} D_{\mu} A_{\alpha} A^{\alpha}+a_{10}(D h)^{\mu} D_{\alpha} A_{\mu} A^{\alpha}+ \\
& +a_{11}(D h)^{\mu}(D A) A_{\mu}+a_{12} D^{\mu} h D_{\mu} A_{\alpha} A^{\alpha}+a_{13} D^{\mu} h(D A) A_{\mu}
\end{aligned}
$$

Not all these terms are independent here. Indeed, it is easy to check that up to terms without derivatives:

$$
\begin{aligned}
D^{\alpha} h^{\mu \nu} D_{\mu} A_{\nu} A_{\alpha} & =h^{\mu \nu} D_{\alpha} A_{\nu} D_{\mu} A^{\alpha}-h^{\mu \nu} D_{\mu} A_{\nu}(D A)+(D h)^{\mu} D_{\alpha} A_{\mu} A^{\alpha} \\
D^{\mu} h D_{\nu} A_{\mu} A^{\nu} & =h(D A)(D A)-h D^{\alpha} A^{\beta} D_{\alpha} A_{\beta}+D^{\mu} h(D A) A_{\mu} \\
D^{\alpha} h^{\mu \nu} D_{\mu} A_{\alpha} A_{\nu} & =(D h)^{\mu}(D A) A_{\mu}+(D h)^{\mu} D_{\alpha} A_{\mu} A^{\alpha}-D^{\alpha} h^{\mu \nu} D_{\mu} A_{\nu} A_{\alpha}
\end{aligned}
$$

Using this freedom and requiring that the Lagrangian will be gauge invariant we finally get:

$$
\mathcal{L}_{h A A}=c_{2} h^{\mu \nu} A_{\mu \alpha} A_{\nu}^{\alpha}-\frac{c_{2}}{4} h A_{\mu \nu}^{2}
$$

while the only non-trivial transformation is:

$$
\delta A_{\mu}=c_{2} \xi^{\nu} A_{\nu \mu}
$$

The result obtained is of course familiar and could seems trivial, but for what follows it is very important that linear approximation does not fix the value of $c_{2}$ coupling constant. In the "normal" massless gravity one expects $c_{2}=2$ but as we will see later on massive theory is possible provided $c_{2}=1$ so that Goldstone field $A_{\mu}$ must have non-canonical $\xi_{\mu}$ transformations.

$h h \varphi$-vertex. Now the most general ansatz for $\xi_{\mu}$ transformations has the form:

$$
\begin{aligned}
\delta h_{\mu \nu} & =c_{1}\left(D_{\mu} \varphi \xi_{\nu}+D_{\nu} \varphi \xi_{\mu}\right)+c_{2} g_{\mu \nu}\left(D_{\alpha} \varphi \xi^{\alpha}\right)+c_{3} \varphi\left(D_{\mu} \xi_{\nu}+D_{\nu} \xi_{\mu}\right)+c_{4} \varphi g_{\mu \nu}(D \xi) \\
\delta \varphi & =c_{5}(D h)_{\mu} \xi^{\mu}+c_{6} D_{\mu} h \xi^{\mu}+c_{7} h^{\mu \nu} D_{\mu} \xi_{\nu}+c_{8} h(D \xi)
\end{aligned}
$$

and using one more time the freedom to make redefinitions:

$\delta h_{\mu \nu}=d_{1}\left[D_{\mu}\left(\varphi \xi_{\nu}\right)+D_{\nu}\left(\varphi \xi_{\mu}\right)\right], \quad h_{\mu \nu} \rightarrow h_{\mu \nu}+s_{1} \varphi h_{\mu \nu}+s_{2} \varphi g_{\mu \nu} h, \quad \varphi \rightarrow \varphi+s_{3} h^{\mu \nu} h_{\mu \nu}+s_{4} h^{2}$ 
one can leave $c_{2}, c_{5}$ and $c_{6}$ as the only non-zero parameters. Then writing most general cubic terms:

$$
\begin{aligned}
\mathcal{L}_{h h \varphi}= & \varphi\left[a_{1} D^{\mu} h^{\alpha \beta} D_{\mu} h_{\alpha \beta}+a_{2} D^{\mu} h^{\alpha \beta} D_{\alpha} h_{\beta \mu}+a_{3}(D h)^{\mu}(D h)_{\mu}+a_{4}(D h)^{\mu} D_{\mu} h+\right. \\
& \left.+a_{5} D^{\mu} h D_{\mu} h\right]+D^{\mu} \varphi\left[a_{6} D_{\mu} h_{\alpha \beta} h^{\alpha \beta}+a_{7} D_{\alpha} h_{\beta \mu} h^{\alpha \beta}+a_{8}(D h)^{\nu} h_{\mu \nu}+\right. \\
& \left.+a_{9} D^{\nu} h h_{\mu \nu}+a_{10} D_{\mu} h h+a_{11}(D h)_{\mu} h\right]
\end{aligned}
$$

and using the fact that up to lower derivative terms we have:

$$
\left(a_{2}\right)=\left(a_{3}\right)-\left(a_{7}\right)+\left(a_{8}\right)
$$

one can check that there is no non-trivial solution for such vertex.

$A A \varphi$-vertex. In this case one has to consider $\lambda$ transformations only, so we write:

$$
\delta A_{\mu}=c_{1} \varphi D_{\mu} \lambda+c_{2} D_{\mu} \varphi \lambda \quad \delta \varphi=c_{3}(D A) \lambda+c_{4} A^{\mu} D_{\mu} \lambda
$$

Possible field and parameter $\lambda$ redefinitions

$$
\delta A_{\mu}=d_{1} D_{\mu}(\varphi \lambda), \quad A_{\mu} \rightarrow A_{\mu}+s_{1} \varphi A_{\mu}, \quad \varphi \rightarrow \varphi+s_{2} A_{\mu}^{2}
$$

allows one to leave $c_{3}$ as the only non-zero parameter. Then considering the most general cubic terms:

$$
\begin{aligned}
\mathcal{L}_{\varphi A A}= & \varphi\left[a_{1} D^{\mu} A^{\nu} D_{\mu} A_{\nu}+a_{2} D^{\mu} A^{\nu} D_{\nu} A_{\mu}+a_{3}(D A)(D A)\right]+ \\
& +D^{\mu} \varphi\left[a_{4} D_{\mu} A_{\nu} A^{\nu}+a_{5}(D A) A_{\mu}\right]
\end{aligned}
$$

and taking into account that

$$
\varphi D^{\mu} A^{\nu} D_{\nu} A_{\mu}=\varphi(D A)(D A)+D^{\mu} \varphi(D A) A_{\mu}-D^{\mu} D_{\nu} A_{\mu} A^{\nu}
$$

we obtain the following simple Lagrangian

$$
\mathcal{L}_{\varphi A A}=a_{0} \varphi A_{\mu \nu}^{2}
$$

which is trivially gauge invariant.

$h \varphi \varphi$-vertex. Here the only possible terms for $\xi_{\mu}$ transformations are:

$$
\delta \varphi=c_{3} D_{\mu} \varphi \xi^{\mu}+c_{4} \varphi(D \xi)
$$

Moreover, using field redefinition

$$
\varphi \rightarrow \varphi+s_{1} h \varphi
$$

we can leave $c_{3}$ as the only non-zero parameter. Then the requirement that the Lagrangian

$$
\mathcal{L}_{h \varphi \varphi}=a_{1} h^{\mu \nu} D_{\mu} \varphi D_{\nu} \varphi+a_{2} h D^{\mu} \varphi D_{\mu} \varphi+a_{3}(D h)^{\mu} D_{\mu} \varphi \varphi+a_{4} D^{\mu} h D_{\mu} \varphi \varphi
$$

will be gauge invariant gives:

$$
a_{1}=-\frac{2 c_{3}(d-1)}{d-2} . \quad a_{2}=\frac{c_{3}(d-1)}{d-2}, \quad a_{3}=-a_{4}
$$


In this, the arbitrary parameter $a_{3}$ is related with one more field redefinition (which does not change the structure of gauge transformations)

$$
h_{\mu \nu} \rightarrow h_{\mu \nu}+s_{2} g_{\mu \nu} \varphi^{2}
$$

So without lost of generality we can set $a_{3}=0$ and obtain:

$$
\begin{aligned}
\mathcal{L}_{h \varphi \varphi} & =\frac{2(d-1)}{d-2}\left[-c_{3} h^{\mu \nu} D_{\mu} \varphi D_{\nu} \varphi+\frac{c_{3}}{2} h(D \varphi)^{2}\right] \\
\delta \varphi & =c_{3} \xi^{\mu} D_{\mu} \varphi
\end{aligned}
$$

Again this result could seems trivial but it is important that coupling constant $c_{3}$ is not fixed yet.

$\varphi \varphi \varphi$-vertex. In this last and simplest case we have only one possible term in the Lagrangian, no non-trivial gauge transformations and one possible field redefinition:

$$
\mathcal{L}_{\varphi^{3}}=a_{1} \varphi D^{\mu} \varphi D_{\mu} \varphi, \quad \varphi \rightarrow \varphi+s_{1} \varphi^{2}
$$

showing that this vertex is trivial one.

Let us collect together all the pieces obtained so far. Total Lagrangian with two derivatives:

$$
\begin{aligned}
\mathcal{L}_{0}= & \mathcal{L}_{h h h}+c_{2} h^{\mu \nu} A_{\mu \alpha} A_{\nu}{ }^{\alpha}-\frac{c_{2}}{4} h A_{\mu \nu}{ }^{2}+a_{0} \varphi A_{\mu \nu}{ }^{2}+ \\
& +\frac{2(d-1)}{d-2}\left[-c_{3} h^{\mu \nu} D_{\mu} \varphi D_{\nu} \varphi+\frac{c_{3}}{2} h(D \varphi)^{2}\right]
\end{aligned}
$$

while the only non-trivial gauge transformations look like:

$$
\begin{aligned}
\delta h_{\mu \nu} & =2\left[\xi^{\alpha} D_{\alpha} h_{\mu \nu}+D_{\mu} \xi^{\alpha} h_{\alpha \nu}+D_{\nu} \xi^{\alpha} h_{\alpha \mu}\right] \\
\delta A_{\mu} & =c_{2} \xi^{\nu} A_{\nu \mu} \quad \delta \varphi=c_{3} \xi^{\mu} D_{\mu} \varphi
\end{aligned}
$$

Let us stress once again that parameters $c_{2}$ and $c_{3}$ are not fixed yet.

Now we proceed to the next order. Namely, we construct the most general cubic terms with one derivative for the Lagrangian as well as the most general linear terms without derivatives for gauge transformations laws. At this order there are no ambiguities related with field redefinitions so all calculations are completely straightforward. The resulting part of the Lagrangian has the form:

$$
\begin{aligned}
\mathcal{L}_{1}= & m\left[4 h^{\mu \nu}(D h)_{\mu} A_{\nu}-h(D h)_{\mu} A^{\mu}+2 h^{\mu \nu} D_{\mu} h_{\nu \alpha} A^{\alpha}-\right. \\
& \left.-3 h^{\mu \nu} D_{\mu} h A_{\nu}-3 h^{\mu \nu} D_{\alpha} h_{\mu \nu} A^{\alpha}+h D_{\mu} h A^{\mu}\right]+ \\
& +2 c_{0} b_{0} h^{\mu \nu} A_{\mu} D_{\nu} \varphi-c_{0} b_{0} h(A D \varphi)+b_{0} m A^{\mu} D_{\mu} \varphi \varphi
\end{aligned}
$$

while additional terms to the gauge transformations are:

$$
\begin{aligned}
\delta h_{\mu \nu} & =m\left[A_{\mu} \xi_{\nu}+A_{\nu} \xi_{\mu}-\frac{4}{d-2} g_{\mu \nu}(A \xi)+\frac{6-d}{d-2} h_{\mu \nu} \lambda\right] \\
\delta A_{\mu} & =m h_{\mu \nu} \xi^{\nu}+2 a_{0} m \varphi \xi_{\mu} \quad \delta \varphi=-c_{0} c_{3}(A \xi)-\frac{m c_{3}}{2} \varphi \lambda
\end{aligned}
$$


Here $b_{0}=\frac{2 c_{3}(d-1)}{d-2}, a_{0}=\frac{m(d-4)}{2 c_{0}(d-2)}, c_{2}=1$ and $c_{3}=1-\frac{m^{2}(d-4)}{2 c_{0}^{2}(d-2)}$. As could easily be seen from these formulas, in particular from the expressions for the $a_{0}$ and $c_{3}$ parameters, in arbitrary space-time dimension $d$ general solution exists for $c_{0} \neq 0$ only. But in $d=4$ dimensions there exists another solution with $c_{0}=0$ then scalar field $\varphi$ completely decouples which corresponds to the self-interaction for partially massless gravity. This fact is clearly connected with the conformal invariance of partially massless theories namely in $d=4$ dimensions [37]. Now we proceed by considering general solution with $c_{0} \neq 0$ in arbitrary space-time dimension but we will comment on partially massless gravity at the end of this section.

The last but not least part of our calculations deals with cubic terms without derivatives in the Lagrangian. The most general form for these terms looks like:

$$
\begin{aligned}
\mathcal{L}_{0}= & b_{1} h^{\mu \nu} h_{\nu \alpha} h^{\alpha \mu}+b_{2} h h^{\mu \nu} h_{\mu \nu}+b_{3} h^{3}+b_{4} \varphi h^{\mu \nu} h_{\mu \nu}+b_{5} \varphi h^{2}+ \\
& +b_{6} \varphi^{2} h+b_{7} \varphi^{3}+b_{8} h^{\mu \nu} A_{\mu} A_{\nu}+b_{9} h A_{\mu}{ }^{2}+b_{10} \varphi A_{\mu}{ }^{2}
\end{aligned}
$$

There are no any new terms for the gauge transformations here, so the gauge invariance must be achieved with the gauge transformations obtained at the previous orders. Indeed, it turns out to be possible and gives:

$$
\begin{gathered}
b_{1}=m^{2}+\frac{(7 d-16) \kappa}{6}, \quad b_{2}=-\frac{5 m^{2}}{4}-\frac{(3 d-7) \kappa}{2}, \quad b_{3}=\frac{m^{2}}{4}+\frac{(2 d-5) \kappa}{6}, \\
b_{4}=-2 b_{5}=\frac{2 c_{0} m(d-1)}{d-2}-\frac{(d-4) m^{3}}{2 c_{0}(d-2)}, \quad b_{6}=\frac{\left(d^{2}+5 d-6\right) m^{2}}{2(d-2)^{2}}-\frac{(3 d-2)(d-4) m^{4}}{4 c_{0}^{2}(d-2)^{2}} \\
b_{7}=2 d c_{0}^{2}\left(d^{2}-11 d+10\right)+\frac{d(d+2)(d-4) m^{5}}{6 c_{0}^{3}(d-2)^{3}} \\
b_{8}=m^{2}-2(d-1) \kappa, \quad b_{9}=\frac{m^{2}}{2}+(d-1) \kappa, \quad b_{10}=-\frac{2 c_{0}(d-1) m}{d-2}+\frac{(d-4) m^{3}}{c_{0}(d-2)}
\end{gathered}
$$

The dependence of these coefficients on the space-time dimension $d$ and cosmological term $\kappa$ (recall that $\left.c_{0}=\sqrt{m^{2}+(d-2) \kappa}\right)$ appears to be rather complicated so let us give explicit expression for non-derivative terms in $d=4$ (any way this dimension is special for us)

$$
\begin{aligned}
\mathcal{L}_{0}= & \left(m^{2}+2 \kappa\right)\left[h^{\mu \nu} h_{\nu \alpha} h^{\alpha \mu}-\frac{5}{4} h h^{\mu \nu} h_{\mu \nu}+\frac{1}{4} h^{3}\right] \\
& +3 c_{0} m \varphi h^{\mu \nu} h_{\mu \nu}-\frac{3 c_{0} m}{2} \varphi h^{2}+\frac{15 m^{2}}{4} \varphi^{2} h-\frac{3 m^{3}}{c_{0}} \varphi^{3}+ \\
& +\left(m^{2}-6 \kappa\right) h^{\mu \nu} A_{\mu} A_{\nu}+\frac{m^{2}+6 \kappa}{2} h_{\mu}{ }^{2}-3 c_{0} m \varphi A_{\mu}{ }^{2}
\end{aligned}
$$

A few comments are in order.

- One of the most important results of our investigation is that possibility to switch on self-interaction in linear approximation crucially depends on the non-canonical form of gauge transformations for vector Goldstone field $A_{\mu}$ (recall, that results of linear 
approximation are model independent up to restriction on the number of derivatives). Gauge transformations we obtained:

$$
\begin{aligned}
\delta h_{\mu \nu} & =\nabla_{\mu} \xi_{\nu}+\nabla_{\nu} \xi_{\mu}+2\left(\xi^{\alpha} \nabla_{\alpha} h_{\mu \nu}+D_{\mu} \xi^{\alpha} h_{\alpha \nu}+D_{\nu} \xi^{\alpha} h_{\mu \alpha}\right) \\
\delta A_{\mu} & =m \xi_{\mu}+\xi^{\alpha} A_{\alpha \mu}+m h_{\mu \nu} \xi^{\nu}
\end{aligned}
$$

resembles very much the situation with massive spin 1 particles which we discussed in section 1:

$$
\delta A_{\mu}^{a}=\partial_{\mu} \xi^{a}-g \varepsilon^{a b c} A_{\mu}{ }^{b} \xi^{c} \quad \delta \varphi^{a}=m \xi^{a}-\frac{g}{2} \varepsilon^{a b c} \varphi^{b} \xi^{c}
$$

So by analogy with spin 1 case one can suggest that there are two possible ways to proceed beyond linear approximation. At first, if one evade an introduction of any additional fields in the model, then this results in highly non-linear theory with higher and higher derivatives. Indeed, we have explicitly checked that one can not construct quadratic approximation without introduction of such higher derivatives terms. On the other hand, one can try to introduce additional fields (analog of Higgs field) in order to restrict the number of derivatives.

- Note that exactly as in the free case all the terms containing vector fields (except kinetic ones) could be "hidden" if one introduce $\lambda$-covariant derivatives:

$$
\begin{aligned}
\nabla_{\mu} h_{\alpha \beta} & =D_{\mu} h_{\alpha \beta}-\frac{2 m}{d-2} A_{\mu} g_{\alpha \beta}-\frac{6-d}{d-2} m A_{\mu} h_{\alpha \beta} \\
\nabla_{\mu} \varphi & =D_{\mu} \varphi-c_{0} A_{\mu}+\frac{m c_{3}}{2} A_{\mu} \varphi
\end{aligned}
$$

- One can always use the $\xi_{\mu}$ and $\lambda$ local gauge transformations to choose the gauge where $A_{\mu}=0$ and $\varphi=0$. In this, the main difference of massive and massless theories are the cubic completion of famous Fierz-Pauli quadratic mass terms:

$$
m^{2}\left[h^{\mu \nu} h_{\nu \alpha} h^{\alpha \mu}-\frac{5}{4} h h^{\mu \nu} h_{\mu \nu}+\frac{1}{4} h^{3}\right]
$$

It is instructive to compare this result with that of [54] (see also [55]) obtained in a very different context. The investigation of [54] shows that allowed cubic terms must be combination of $h^{\mu \nu} h_{\nu \alpha} h^{\alpha}{ }_{\mu}-h h^{\mu \nu} h_{\mu \nu}$ and $h^{\mu \nu} h_{\nu \alpha} h^{\alpha}{ }_{\mu}-\frac{3}{2} h h^{\mu \nu} h_{\mu \nu}+\frac{1}{2} h^{3}$. It is easy to see that our terms correspond to combination with coefficients $\frac{1}{2}$, so at this point our results agree with that of [54].

- In the discussions of possible mass terms for gravity it is very often assumed that the full theory could be the sum of usual massless gravity plus some invariant mass terms, e.g. [17]. If we denote effective metric as $\hat{g}_{\mu \nu}$ and assume that in the lowest approximation $\sqrt{-\hat{g}} \simeq \sqrt{-g}+h$ and $\hat{g}^{\mu \nu} \simeq g^{\mu \nu}-2 h^{\mu \nu}$ then we easily obtain:

$$
-\frac{m^{2}}{2} \sqrt{-\hat{g}} \hat{g}^{\mu \alpha} \hat{g}^{\nu \beta}\left(h_{\mu \nu} h_{\alpha \beta}-h_{\mu \alpha} h_{\nu \beta}\right) \simeq 2 m^{2}\left[h^{\mu \nu} h_{\nu \alpha} h^{\alpha \mu}-\frac{5}{4} h h^{\mu \nu} h_{\mu \nu}+\frac{1}{4} h^{3}\right]
$$

so the structure of cubic terms does not contradict (up to a factor 2) such assumption. Nevertheless, let us stress that in the massive case the gauge transformations of tensor $h_{\mu \nu}$ is changed so it is hard (if at all possible) to represent massive theory as a sum of two parts which are separately gauge invariant. 
- In the massless limit massive spin 2 particle in $(A) d S_{d}$ decompose into massless spin 2 particle and massive spin 1 (or massless spin 1 and spin 0 in flat space). In this, two gauge transformations $\xi_{\mu}$ and $\lambda$ are completely independent and $\lambda$ transformation is just usual gauge transformation of abelian vector field. But then mass $m \neq 0$ the commutator of $\xi_{\mu}$ and $\lambda$ transformations gives:

$$
\begin{aligned}
{[\delta(\lambda), \delta(\xi)] h_{\mu \nu} } & =m\left[D_{\mu}\left(\xi_{\nu} \lambda\right)+D_{\nu}\left(\xi_{\mu} \lambda\right)\right] \\
{[\delta(\lambda), \delta(\xi)] A_{\mu} } & =m^{2} \xi_{\mu} \lambda
\end{aligned}
$$

so if we denote a generator of $\xi_{\mu}$ transformation as $P_{\mu}$ and that of $\lambda$ one as $D$, we get commutation relation for Weyl group $\left[P_{\mu}, D\right] \sim m P_{\mu}$ !

- As we have already mentioned, apart form general solution which exists in arbitrary dimension $d$, in $d S_{4}$ and only in this dimension there exist another solution with $c_{0}=$ $\sqrt{m^{2}+2 \kappa}=0$ which corresponds to self interaction of partially massless spin 2 particle in de Sitter background. In this, scalar field $\varphi$ completely decouples, leaving us with $h_{\mu \nu}$ and $A_{\mu}$ fields (note the absence of $h^{3}$ terms):

$$
\begin{aligned}
\mathcal{L}_{\text {int }}= & \mathcal{L}_{h h h}+h^{\mu \nu} A_{\mu \alpha} A_{\nu}{ }^{\alpha}-\frac{1}{4} h A_{\mu \nu}{ }^{2}+ \\
& +m\left[4 h^{\mu \nu}(D h)_{\mu} A_{\nu}-h(D h)_{\mu} A^{\mu}+2 h^{\mu \nu} D_{\mu} h_{\nu \alpha} A^{\alpha}-\right. \\
& \left.-3 h^{\mu \nu} D_{\mu} h A_{\nu}-3 h^{\mu \nu} D_{\alpha} h_{\mu \nu} A^{\alpha}+h D_{\mu} h A^{\mu}\right]
\end{aligned}
$$

Using local $\xi_{\mu}$ gauge transformation one can always choose the gauge $A_{\mu}=0$. As in the free case, the resulting Lagrangian will still be invariant under $\lambda$ transformation provided we supplement it with the appropriate restoring gauge $A_{\mu}=0 \xi_{\mu}$ transformation. Indeed, let us look at the form of these transformations when $A_{\mu}=0$ :

$$
\begin{aligned}
\delta h_{\mu \nu} & =D_{\mu} \xi_{\nu}+D_{\nu} \xi_{\mu}+m g_{\mu \nu} \lambda+2\left(\xi^{\alpha} D_{\alpha} h_{\mu \nu}+D_{\mu} \xi^{\alpha} h_{\alpha \nu}+D_{\nu} \xi^{\alpha} h_{\alpha \mu}\right)+m h_{\mu \nu} \lambda \\
\delta A_{\mu} & =D_{\mu} \lambda+m \xi_{\mu}+m h_{\mu \nu} \xi^{\nu}
\end{aligned}
$$

Then it is easy to see that compensating gauge transformation has to be:

$$
\xi_{\mu} \simeq-\frac{1}{m}\left[D_{\mu} \lambda+h_{\mu \nu} D^{\nu} \lambda\right]
$$

We have explicitly checked that the Lagrangian for the $h_{\mu \nu}$ field is indeed invariant under such combined $\lambda$ and $\xi_{\mu}$ transformations. In the $A_{\mu}=0$ gauge the Lagrangian looks as the linearization of usual gravity in $d S_{4}$ background but this holds in the linear approximation only. We have checked that it is impossible to proceed with quadratic approximation without introduction of higher derivative terms and/or some other fields.

\section{Interaction with matter}

In this section we investigate possible interactions of massive spin 2 particles with matter fields of lower spins. The strategy will be the same as in the previous section - we construct the most general cubic terms with no more than two derivatives in the Lagrangians and corresponding linear terms with no more than one derivative for gauge transformation laws with the only requirement that the Lagrangian be gauge invariant. 


\subsection{Spin 0}

We start with the simplest case of massive spin 0 particles with the usual free Lagrangian:

$$
\mathcal{L}_{0}=\frac{1}{2}\left(\partial_{\mu} \pi\right)^{2}-\frac{m_{0}^{2}}{2} \pi^{2}
$$

All calculations here are very simple and one can easily check that up to possible field redefinitions an interacting Lagrangian has a form:

$$
\begin{aligned}
\mathcal{L}_{1}= & -\frac{c_{2}}{2}\left[h^{\mu \nu} \partial_{\mu} \pi \partial_{\nu} \pi-\frac{1}{2} h\left(\partial_{\mu} \pi\right)^{2}+\frac{m}{c_{0}} \varphi\left(\partial_{\mu} \pi\right)^{2}\right]- \\
& -\frac{c_{2} m_{0}^{2}}{4}\left[h-4 \frac{m}{c_{0}} \varphi\right] \pi^{2}
\end{aligned}
$$

while gauge transformations for $\pi$ field look like:

$$
\delta \pi=c_{2} \xi^{\mu} \partial_{\mu} \pi
$$

We see that vector field $A_{\mu}$ does not couple at all (because $\pi$ field is inert under $\lambda$ transformations), while coupling of scalar field $\varphi$ clearly shows an ambiguity between flat space and massless limits. Indeed, this coupling depends on $m / c_{0}=m / \sqrt{m^{2}+(d-2) \kappa}$ and we have two different limits. On one hand, we can take a massless limit $m \rightarrow 0$ while keeping cosmological term $\kappa$ small but non-zero. In this, scalar field $\varphi$ completely decouples so that massless limit of massive theory agrees with purely massless theory results. On the other hand, if we take flat space limit $\kappa \rightarrow 0$ while keeping non-zero mass $m$, then the coupling constant for scalar field $\varphi$ tends to 1 and does not depend on mass any more. As a result, in the massless limit scalar field $\varphi$ does not decouple from matter field $\pi$.

\subsection{Spin 1}

Our next example - interaction with massive spin 1 particles. Due to our usage of gauge invariant description this includes massless limit as well. Thus, we introduce two fields vector $B_{\mu}$ and Goldstone scalar $\pi$ and start with the free Lagrangian which has its own gauge invariance:

$$
\begin{aligned}
\mathcal{L}_{0} & =-\frac{1}{4} B_{\mu \nu}{ }^{2}+\frac{1}{2}\left(D_{\mu} \pi\right)^{2}-m_{1} B^{\mu} D_{\mu} \pi+\frac{m_{1}{ }^{2}}{2} B_{\mu}{ }^{2} \\
\delta B_{\mu} & =\partial_{\mu} \tilde{\lambda}, \quad \delta \pi=m_{1} \tilde{\lambda}
\end{aligned}
$$

An investigation of possible $h B B$ and $h \pi \pi$ vertexes with two derivatives goes exactly the same way as that of $h A A$ and $h \varphi \varphi$ vertexes in the previous section, so we will not repeat these calculations here and write the corresponding part of Lagrangian:

$$
\begin{aligned}
\mathcal{L}_{1}= & \frac{c_{1}}{2} h^{\mu \nu} B_{\mu \alpha} B_{\nu \alpha}-\frac{c_{1}}{8} h B_{\mu \nu}{ }^{2}-\frac{c_{2}}{2} h^{\mu \nu} D_{\mu} \pi D_{\nu} \pi+\frac{c_{2}}{4} h\left(D_{\mu} \pi\right)^{2}+ \\
& +a_{0} \varphi\left(D_{\mu} \pi\right)^{2}+a_{1} \varphi B_{\mu \nu}{ }^{2}+a_{2} \pi A^{\mu \nu} B_{\mu \nu}
\end{aligned}
$$


which is invariant under the following transformations:

$$
\delta B_{\mu}=c_{1} \xi^{\nu} B_{\nu \mu}, \quad \delta \pi=c_{2} \xi^{\mu} D_{\mu} \pi
$$

Now we proceed by adding all possible lower derivatives terms to the Lagrangian and corresponding terms to gauge transformations. This procedure results in:

$$
\begin{aligned}
\mathcal{L}_{2}= & m_{1} c_{1}\left[h^{\mu \nu} B_{\mu} D_{\nu} \pi-\frac{1}{2} h(B D \pi)+\frac{m}{c_{0}} \varphi(B D \pi)\right]- \\
& -\frac{m_{1}{ }^{2} c_{1}}{2}\left[h^{\mu \nu} B_{\mu} B_{\nu}-\frac{1}{2} h B_{\mu}{ }^{2}+\frac{m}{c_{0}} \varphi B_{\mu}{ }^{2}\right]
\end{aligned}
$$

with the only new term in gauge transformations:

$$
\delta^{\prime} \pi=-c_{1} m_{1} \xi^{\mu} B_{\mu}
$$

In this, gauge invariance requires $c_{1}=c_{2}, a_{0}=\frac{m}{c_{0}}, a_{1}=\frac{c_{1} m(d-4)}{4 c_{0}(d-2)}, a_{2}=0$. Once again, our results show an ambiguity betwen flat space and massless limits. Only for massless vector field in $d=4$ dimensions this ambiguity is absent.

\subsection{Spin $1 / 2$}

Our last example - interaction with massive spin 1/2 particles (recall that we prefer to work with Majorana spinors). As is well known, to describe spinor fields living in curved background one has to use first order formalism in terms of "tetrad" $e_{\mu}{ }^{a}$ and Lorentz connection so that $\gamma_{\mu}=e_{\mu a} \gamma^{a}$ and

$$
\left[D_{\mu}, D_{\nu}\right] \chi=\frac{1}{4} R_{\mu \nu}^{a b} \sigma_{a b} \chi=\frac{\kappa}{2} \sigma_{\mu \nu} \chi
$$

In this, to describe an interaction of spinor fields with our massive spin 2 particles it is also convenient to use first order formulation of such particles [56] in terms of three pairs of fields: $\left(e_{\mu a}, \omega_{\mu}^{a b}\right),\left(A_{\mu}, F^{a b}\right)$ and $\left(\varphi, \pi^{a}\right)$. We will not reproduce Lagrangian for such formulation here (it could easily be found in [56]) because all we need here is the structure of gauge transformations:

$$
\begin{aligned}
\delta h_{\mu a} & =D_{\mu} \xi_{a}+\eta_{\mu a}+\frac{m}{2} e_{\mu a} \lambda \\
\delta \omega_{\mu}^{a b} & =D_{\mu} \eta^{a b}-\frac{c_{0}^{2}}{2}\left(e_{\mu}{ }^{a} \xi^{b}-e_{\mu}{ }^{b} \xi^{a}\right) \\
\delta A_{\mu} & =D_{\mu} \lambda+m \xi_{\mu} \quad \delta \varphi=c_{0} \lambda
\end{aligned}
$$

where apart from $\xi^{a}$ and $\lambda$ gauge transformations we have now one more transformation with parameter $\eta_{a b}=-\eta_{b a}$. Again we start with the free Lagrangian for massive spinor $\chi$ :

$$
\mathcal{L}_{0}=\frac{i}{2} \bar{\chi} \gamma^{\mu} D_{\mu} \chi-\frac{m_{1 / 2}}{2} \bar{\chi} \chi
$$

and construct the most general cubic terms (this time with no more than one derivative) compatible with all gauge symmetries. We obtain for interaction Lagrangian:

$$
\begin{aligned}
\mathcal{L}_{1}= & -\frac{i}{2} \bar{\chi} h^{\mu \nu} \gamma_{\mu} D_{\nu} \chi+\frac{i}{2} \bar{\chi} h \gamma^{\mu} D_{\mu} \chi-\frac{i}{8} \bar{\chi} \gamma^{\mu} \omega_{\mu}{ }^{a b} \sigma_{a b} \chi- \\
& -\frac{m_{1 / 2}}{2}\left(h-\frac{m}{2 c_{0}} \varphi\right) \bar{\chi} \chi
\end{aligned}
$$


while for gauge transformations we get:

$$
\delta \chi=\xi^{\mu} D_{\mu} \chi-\frac{1}{4}(\sigma \eta) \chi-\frac{3 m}{4} \lambda \chi
$$

In this case an ambiguity between flat space and massless limits exists for massive $m_{1 / 2} \neq 0$ spinor field only, while the results for massless spinor agree with that of purely massless gravity.

\section{$5 \quad$ Interaction with gravity}

There exist well known difficulties one faces in any attempt to construct interacting theory for a collection of massless spin 2 particles [38, 39, 40, 41, 42]. For example, for the case of just two massless spin 2 particles there are only two possibilities. One of them corresponds to two copies of usual gravities completely independent of each other, while the other possibility which does have interaction requires that one of these particles has wrong sign of kinetic terms and be a ghost. Moreover, there are examples of theories of such kind, where one of spin 2 particles is massive, coming from higher derivative gravity models e.g. [57]. Also, as is well known, there are examples of consistent theories with infinite number of massive spin 2 particles in Kaluza-Klein models, but it could be shown [45, 48] that it does not contradict with general results of [38, 39].

But all this still leaves a possibility to construct a consistent theory where one massless spin 2 particle interacts with one or several massive spin 2 ones. In this section we investigate the case of one massless and one massive particles. In this, there are two possible cubic vertexes (besides self-interaction). We have checked (though we will not present details of these calculations here) that it is impossible to construct linear approximation with two massless and one massive particles (once again up to the restriction on the number of derivatives). As for the other case, i.e. interaction of massive spin 2 particles with usual gravity, it is not hard to constract general covariant vertex with arbitrary number of massless fields but still bi-linear in massive fields, as we are going to demonstrate.

First of all, let us note that in this section metric $g_{\mu \nu}$ is not just a fixed background any more, but it is a dynamical field with its own equation of motion:

$$
R_{\mu \nu}-\frac{1}{2} g_{\mu \nu} R+\frac{\kappa(d-1)(d-2)}{2} g_{\mu \nu}=0
$$

As usual in gravity, we will assume that connection is metric compatible $D_{\alpha} g_{\mu \nu}=0$ and we have usual identities:

$$
\begin{aligned}
D^{\mu} R_{\mu \nu, \alpha \beta} & =D_{\alpha} R_{\beta \nu}-D_{\beta} R_{\alpha \nu} \\
D^{\mu} R_{\mu \nu} & =\frac{1}{2} D_{\nu} R
\end{aligned}
$$

As in the case of massive particle living in constant curvature background, it is convenient to organize the calculations by the number of derivatives. So we start with the sum of kinetic 
terms of our three fields $h_{\mu \nu}, A_{\mu}$ and $\varphi$ :

$$
\begin{aligned}
\mathcal{L}_{2}= & \frac{1}{2} D^{\alpha} h^{\mu \nu} D_{\alpha} h_{\mu \nu}-D^{\alpha} h^{\mu \nu} D_{\mu} h_{\nu \alpha}+(D h)^{\mu} D_{\mu} h-\frac{1}{2} D^{\mu} h D_{\mu} h- \\
& -\frac{1}{2}\left(D_{\mu} A_{\nu}-D_{\nu} A_{\mu}\right)^{2}+\frac{2(d-1)}{(d-2)} D^{\mu} \varphi D_{\mu} \varphi
\end{aligned}
$$

and corresponding gauge transformations with one derivative:

$$
\delta_{1} h_{\mu \nu}=D_{\mu} \xi_{\nu}+D_{\nu} \xi_{\mu}, \quad \delta_{1} A_{\mu}=D_{\mu} \lambda
$$

Covariant derivatives do not commute and, as a result, this Lagrangian is not invariant under these gauge transformations. But gauge invariance could be restored (up to lower derivative terms we will take into account later) if one adds to the Lagrangian:

$$
\Delta \mathcal{L}=-2 R^{\mu \nu} h_{\mu \alpha} h_{\nu \alpha}+R^{\mu \nu} h_{\mu \nu} h+\frac{1}{2} R h^{\alpha \beta} h_{\alpha \beta}-\frac{1}{4} R h^{2}
$$

and requires that metric $g_{\mu \nu}$ has non-trivial transformation

$$
\delta_{1} g_{\alpha \beta}=2\left(D_{\mu} h_{\alpha \beta}-D_{\alpha} h_{\beta \mu}-D_{\beta} h_{\alpha \mu}\right) \xi^{\mu}
$$

Now we proceed with lower derivative terms. The part of the Lagrangian with one derivatives turns out to be:

$$
\mathcal{L}_{1}=2 m\left[h^{\mu \nu} D_{\mu} A_{\nu}-h(D A)\right]-\frac{4(d-1) c_{0}}{(d-2)} A^{\mu} D_{\mu} \varphi
$$

while corrections to gauge transformation look like:

$$
\delta_{0} h_{\mu \nu}=\frac{2 m}{(d-2)} g_{\mu \nu} \lambda, \quad \delta_{0} A_{\mu}=m \xi_{\mu}, \quad \delta_{0} \varphi=c_{0} \lambda
$$

Also this requires additional modification of $g_{\mu \nu}$ gauge transformations:

$$
\delta_{0} g_{\alpha \beta}=2 m\left[A_{\alpha} \xi_{\beta}+A_{\beta} \xi_{\alpha}-\frac{2}{(d-2)} g_{\alpha \beta}(A \xi)\right]-\frac{2 m(d-4)}{(d-2)} h_{\alpha \beta} \lambda
$$

At last, we must add possible terms without derivatives, the most general form being:

$$
\mathcal{L}_{0}=-\frac{b_{1}}{2} h^{\mu \nu} h_{\mu \nu}+\frac{b_{2}}{2} h^{2}+b_{3} h \varphi+b_{4} \varphi^{2}+b_{5} A_{\mu}{ }^{2}
$$

There are no any additional corrections to gauge transformations at this level, so the gauge invariance must be achieved with the ones we already have. And indeed it turns out to be possible giving:

$$
\begin{gathered}
b_{1}=m^{2}+\kappa(d-1)(d-2), \quad b_{2}=m^{2}+\frac{\kappa}{2}(d-1)(d-2) \\
b_{3}=-\frac{2(d-1) m c_{0}}{(d-2)}, \quad b_{4}=\frac{2 m^{2} d(d-1)}{(d-2)^{2}}, \quad b_{5}=2 \kappa(d-1)
\end{gathered}
$$


This time also there is a possibility greatly simplify all the expressions by introduction of $\lambda$-covariant derivatives:

$$
\nabla_{\mu} h_{\alpha \beta}=D_{\mu} h_{\alpha \beta}-\frac{2 m}{(d-2)} A_{\mu} g_{\alpha \beta}, \quad \nabla_{\mu} \varphi=D_{\mu} \varphi-c_{0} A_{\mu}
$$

In this, total Lagrangian could be rewritten in the form:

$$
\begin{aligned}
\mathcal{L}= & \frac{1}{2} \nabla^{\alpha} h^{\mu \nu} \nabla_{\alpha} h_{\mu \nu}-\nabla^{\alpha} h^{\mu \nu} \nabla_{\mu} h_{\nu \alpha}+(\nabla h)^{\mu} \nabla_{\mu} h-\frac{1}{2} \nabla^{\mu} h \nabla_{\mu} h- \\
& -\frac{1}{2}\left(D_{\mu} A_{\nu}-D_{\nu} A_{\mu}\right)^{2}+\frac{2(d-1)}{(d-2)} \nabla^{\mu} \varphi \nabla_{\mu} \varphi- \\
& -2 R^{\mu \nu} h_{\mu \alpha} h_{\nu \alpha}+R^{\mu \nu} h_{\mu \nu} h+\frac{1}{2} R h^{\alpha \beta} h_{\alpha \beta}-\frac{1}{4} R h^{2}- \\
& -\frac{b_{1}}{2} h^{\mu \nu} h_{\mu \nu}+\frac{b_{2}}{2} h^{2}+b_{3} h \varphi+b_{4} \varphi^{2}
\end{aligned}
$$

while gauge transformations for the metric field $g_{\mu \nu}$ look like:

$$
\delta g_{\alpha \beta}=2\left(\nabla_{\mu} h_{\alpha \beta}-\nabla_{\alpha} h_{\beta \mu}-\nabla_{\beta} h_{\alpha \mu}\right) \xi^{\mu}+\frac{2 m(d-4)}{(d-2)}\left[g_{\alpha \beta}(A \xi)-h_{\alpha \beta} \lambda\right]
$$

Thus, in this approximation an interaction of massive spin 2 particles with gravity exists in any space-time dimension $d \geq 3$ (though from the last equation one can see that $d=4$ case is also special here). In particular, nothing prevent us to consider the $c_{0} \rightarrow 0$ limit, i.e. interaction of partially massless spin 2 particle with gravity. But as in the case of self-interaction, as we have explicitly checked, if one tries to proceed with terms quartic in massive fields than one will find that higher derivatives interactions and/or some additional fields are necessary. It is instructive to compare our results here with the investigations of massive spin 2 particle in gravitational background [11, 12, 13. In general, results are similar, but the structure of $R h h$ terms is slightly differrent.

\section{Conclusion}

We hope that the main lesson from our paper is that constructive approach based on gauge invariant description of massive high spin particles does allow one efficiently investigate possible interactions of such particles. It is important that due to peculiarity of linear approximation the results obtained for any particle are completely model independent and do not depend on the presence of any other fields in the theory. In particular, an impossibility to construct an interaction in linear approximation means that such interaction does not exist at all. One of the examples of such "no-go" results is the absence of self-interaction for partially massless spin 2 particles in $d \neq 4$ dimensions.

One of the striking and unexpected results is that the existence of self-interaction for massive spin 2 particles crucially depends on non-canonical form of gauge transformations for Goldstone $A_{\mu}$ field. By analogy with massive spin 1 case, one can assume that in full interacting theory (if it exists at all) one must deal with non-linear realization of $\xi_{\mu}$ symmetry 
with higher and higher derivatives. We are not aware on any works on non-linear realization of space-time symmetries where something similar appears.

In the investigation of the massive spin 2 particle interacting with matter (i.e. spin 0,1 , and $1 / 2$ particles) out results clearly show the ambiguity betwen flat space and massless limits which reveals itself through the dependence of scalar Goldstone field $\varphi$ coupling constant on mass and cosmological constant. Let us stress once again that this results are also model independent.

Throughout the paper we restrict ourselves with interaction terms with no more than two derivatives in the Lagrangians (and correspondingly no more than one derivative in gauge transformations). But many of our results clearly show that to construct full interacting theory beyond linear approximations one unavoidably will have to introduce higher derivatives

interactions. But such higher derivatives interactions could, in principle, change the results obtained here for linear approximation. This question deserves further study.

\section{References}

[1] V. I. Ogievetsky, I. V. Polubarinov "Interacting field of spin 2 and the Einstein equations", Ann. Phys. 35 (1965) 167.

[2] J. Fang, C. Fronsdal "Deformations of gauge groups. Gravitation", J. Math. Phys. 20 (1979) 2264.

[3] K. A. Milton, L. F. Urrutia, R. J. Finkelstein "Constructive approach to supergravity", Gen. Rel. Grav. 12 (1980) 67.

[4] R. M. Wald "Spin-two fields and general covariance", Phys. Rev. D33 (1986) 3613.

[5] I. L. Buchbinder, A. Fotopoulos, A. C. Petkou, M. Tsulaia "Constructing the Cubic Interaction Vertex of Higher Spin Gauge Fields", hep-th/0609082.

[6] C. R. Hagen, L. P. S. Singh "Search for consistent interactions of the Rarita-Schwinger field", Phys. Rev. D26 (1982) 393.

[7] S. Ferrara, M. Porrati, V. L. Telegdi " $g=2$ as the natural value of the tree-level gyromagnetic ratio of elementary particles", Phys. Rev. D46 (1992) 3529.

[8] A. Cucchieri, S. Deser, M. Porrati "Tree-level unitarity constraints on the gravitational couplings of higher-spin massive fields", Phys. Rev. D51 (1995) 4543, hep-th/9408073.

[9] S. Deser, V. Pascalutsa, A. Waldron "Massive spin 3/2 electrodynamics", Phys. Rev. D62 (2000) 105031, hep-th/0003011.

[10] S. Deser, A. Waldron "Inconsistencies of massive charged gravitating higher spins", Nucl. Phys, B631 (2002) 369, hep-th/0112182.

[11] I. L. Buchbinder, V. A. Krykhtin, V. D. Pershin "On Consistent Equations for Massive Spin-2 Field Coupled to Gravity in String Theory", Phys. Lett. B466 (1999) 216, hep-th/9908028. 
[12] I. L. Buchbinder, D. M. Gitman, V. A. Krykhtin, V. D. Pershin "Equations of Motion for Massive Spin 2 Field Coupled to Gravity", Nucl. Phys. B584 (2000) 615, hep-th/9910188.

[13] I. L. Buchbinder, D. M. Gitman, V. D. Pershin "Causality of Massive Spin 2 Field in External Gravity", Phys. Lett. B492 (2000) 161, hep-th/0006144.

[14] Yu. M. Zinoviev "Gauge invariant description of massive high spin particles", preprint IHEP 83-91, Protvino, 1983.

[15] S. M. Klishevich, Yu. M. Zinoviev "On electromagnetic interaction of massive spin-2 particle", Phys. Atom. Nucl. 61 (1998) 1527, hep-th/9708150.

[16] Yu. M. Zinoviev "On Massive High Spin Particles in (A)dS", hep-th/0108192.

[17] N. Arkani-Hamed, H. Georgi, M. D. Schwartz "Effective Field Theory for Massive Gravitons and Gravity in Theory Space", Ann. Phys. 305 (2003) 96, hep-th/0210184.

[18] S. Hamamoto "Possible Nonlinear Completion of Massive Gravity", Prog. Theor. Phys. 114 (2006) 1261, hep-th/0505194.

[19] M. Bianchi, P. J. Heslop, F. Riccioni "More on La Grande Bouffe", JHEP 08 (2005) 088, hep-th/0504156.

[20] K. Hallowell, A. Waldron "Constant Curvature Algebras and Higher Spin Action Generating Functions", Nucl. Phys. B724 (2005) 453, hep-th/0505255.

[21] I. L. Buchbinder, V. A. Krykhtin "Gauge invariant Lagrangian construction for massive bosonic higher spin fields in D dimensions", Nucl. Phys. B727 (2005) 537, hep-th/0505092.

[22] I. L. Buchbinder, V. A. Krykhtin, P. M. Lavrov "Gauge invariant Lagrangian formulation of higher spin massive bosonic field theory in AdS space", hep-th/0608005.

[23] R. R. Metsaev "Gauge invariant formulation of massive totally symmetric fermionic fields in (A)dS space", hep-th/0609029.

[24] L. D. Faddeev, A. A. Slavnov "Invariant perturbation theory for non-linear chiral Lagrangians", Teor. Mat. Fiz. 8 (1971) 297.

[25] D. G. Boulware, S. Deser "Can gravitation have a finite range?" Phys. Rev. D6 (1972) 3368.

[26] S. V. Babak, L. P. Grishchuk "Finite-Range Gravity and Its Role in Gravitational Waves, Black Holes and Cosmology", Int. J. Mod. Phys. D12 (2003) 1905, gr-qc/0209006.

[27] A. N. Petrov "The field theoretical formulation of general relativity and gravity with non-zero masses of gravitons", gr-qc/0505058. 
[28] V. I. Zakharov "Linearized gravitation theory and graviton mass", JEThP Letters 13 (1970) 312.

[29] H. van Dam, M. Veltman "On the mass of graviton", Gen. Rel. Grav. 3 (1972) 215.

[30] I. I. Kogan, S. Mouslopoulos, A. Papazoglou "The $m \rightarrow 0$ limit for massive graviton in $d S_{4}$ and $A d S_{4}$. How to circumvent the van Dam-Veltman-Zakharov discontinuity", Phys. Lett. B503 (2001) 173, hep-th/0011138.

[31] M. Porrati "No Van Dam-Veltman-Zakharov Discontinuity in Ads Space", Phys. Lett. B498 (2001) 92, hep-th/0011152.

[32] C. Deffayet, G. Dvali, G. Gabadadze, A. I. Vainstein "Nonperturbative continuity in graviton mass versus perturbative discontinuity", Phys. Rev. D65 (2002) 044026, hep-th/0106001.

[33] S. Deser, A. Waldron "Gauge Invariance and Phases of Massive Higher Spins in (A)dS", Phys. Rev. Lett. 87 (2001) 031601, hep-th/0102166.

[34] S. Deser, A. Waldron "Partial Masslessness of Higher Spins in (A)dS", Nucl. Phys. B607 (2001) 577, hep-th/0103198.

[35] S. Deser, A. Waldron "Null Propagation of Partially Massless Higher Spins in (A)dS and Cosmological Constant Speculations", Phys. Lett. B513 (2001) 137, hep-th/0105181.

[36] E. D. Skvortsov, M. A. Vasiliev "Geometric Formulation for Partially Massless Fields", hep-th/0601095.

[37] S. Deser, A. Waldron "Conformal Invariance of Partially Massless Higher Spins", hep-th/0408155.

[38] R. M. Wald "A new type of gauge invariance for a collection of massless spin-2 fields: I. Existence and uniqueness", Class. Quant. Grav. 4 (1987) 1267.

[39] R. M. Wald "A new type of gauge invariance for a collection of massless spin-2 fields: II. Geometrical interpretation", Class. Quant. Grav. 4 (1987) 1279.

[40] N. Boulanger, T. Damour, L. Gualtieri, M. Henneaux "Inconsistency of interacting, multi-graviton theories", Nucl. Phys. B597 (2001) 127, hep-th/0007220.

[41] N. Boulanger "Multi-graviton theories : yes-go and no-go results", Fortsch. Phys. 50 (2002) 858, hep-th/0111216.

[42] S. C. Anco "On multi-graviton and multi-gravitino gauge theories", Class. Quant. Grav. 19 (2003) 6445, gr-qc/0303033.

[43] L. Dolan, M. J. Duff "Kac-Moody Symmetries of Kaluza-Klein Theories", Phys. Rev. Lett. 52 (1984) 14. 
[44] C. S. Aulakh, D. Sahdev "The Infifnite-dimensional Guage Structure of Kaluza-Klein Theories I. D = $1+4 "$, Phys. Lett. B164 (1985) 293.

[45] M. Reuter "Consistent Interaction for Infinitely Many Massless Spin-Two Fields by Dimensional Reduction", Phys. Lett. B205 (1988) 511.

[46] Y. M. Cho, S. W. Zoh "Explicit construction of massive spin-two fields in Kaluza-Klein theory", Phys. Rev. D46 (1992) 2290.

[47] V. A. Tsokur, Yu. M. Zinoviev " $N=2$ Supergravity Models with Gauge Kac-Moody Groups", hep-th/9607034.

[48] O. Hohm "On the infinite-dimensional spin-2 symmetries in Kaluza-Klein theories", Phys. Rev. D73 (2006) 044003, hep-th/0511165.

[49] E. Alvarez, D. Blas, J. Garriga, E. Verdaguer "Transverse Fierz-Pauli symmetry", Nucl. Phys. B756 (2006) 148, hep-th/0606019.

[50] A. H. Chamseddine "Spontaneous Symmetry Breaking for Massive Spin-2 Interacting with Gravity", Phys. Lett. B557 (2003) 247, hep-th/0301014.

[51] S. Deser, A. Waldron "Partially Massless Spin 2 Electrodynamics", hep-th/0609113.

[52] D. R. Grigore, G. Scharf "Massive gravity as a quantum gauge theory", Gen. Rel. Grav. 37 (2005) 1075, hep-th/0404157.

[53] T. Padmanabhan "From Gravitons to Gravity: Myths and Reality", gr-qc/0409089.

[54] P. Creminelli, A. Nicolis, M. Papucci, E. Trincherini "Ghosts in Massive Gravity", JHEP 0509 (2005) 003, hep-th/0505147.

[55] C. Deffayet, J-W. Rombouts "Ghosts, Strong Coupling and Accidental Symmetries in Massive Gravity", Phys. Rev. D72 (2005) 044003, gr-qc/0505134.

[56] Yu. M. Zinoviev "First Order Formalism for Massive Mixed Symmetry Tensor Fields in Minkowski and (A)dS Spaces", hep-th/0306292.

[57] G. Magnano, L. M. Sokolowski "Nonlinear massive spin-two field generated by higher derivative gravity", Ann. Phys. 306 (2003) 1, gr-qc/0209022. 